\title{
Strain-induced structural instability in FeRh
}

\author{
Ulrich Aschauer, ${ }^{1,2}$ Roisin Braddell, ${ }^{1}$ Sonia A. Brechbühl, ${ }^{1}$ Peter M. Derlet, ${ }^{3}$ and Nicola A. Spaldin ${ }^{1}$ \\ ${ }^{1}$ Materials Theory, ETH Zurich, Wolfgang-Pauli-Strasse 27, CH-8093 Zürich, Switzerland \\ ${ }^{2}$ Department of Chemistry and Biochemistry, University of Bern, Freiestrasse 3, CH-3012 Bern, Switzerland \\ ${ }^{3}$ Condensed Matter Theory Group, Paul Scherrer Institute, CH-5232 Villigen PSI, Switzerland
}

(Received 13 March 2016; revised manuscript received 15 June 2016; published 12 July 2016)

\begin{abstract}
We perform density functional calculations to investigate the structure of the intermetallic alloy FeRh under epitaxial strain. Bulk FeRh exhibits a metamagnetic transition from a low-temperature antiferromagnetic (AFM) phase to a ferromagnetic phase at $350 \mathrm{~K}$, and its strain dependence is of interest for tuning the transition temperature to the room-temperature operating conditions of typical memory devices. We find an unusually strong dependence of the structural energetics on the choice of exchange-correlation functional, with the usual local density approximation yielding the wrong ground-state structure, and generalized gradient (GGA) extensions being in better agreement with the bulk experimental structure. Using the GGA we show the existence of a metastable face-centered-cubic-like AFM structure that is reached from the ground-state body-centered-cubic-like AFM structure by following the epitaxial Bain path. We show that the behavior is well described using nonlinear elasticity theory, which captures the softening and eventual sign change of the orthorhombic shear modulus under compressive strain, consistent with this structural instability. Finally, we predict the existence of an additional unit-cell-doubling lattice instability, which should be observable at low temperature.
\end{abstract}

DOI: 10.1103/PhysRevB.94.014109

\section{INTRODUCTION}

The intermetallic compound FeRh exhibits an unusual first-order phase transition from an antiferromagnetic (AFM) structure at low temperature to a ferromagnetic (FM) structure above roughly $350 \mathrm{~K}[1,2]$. The phase transition is isostructural, with both phases having the $\mathrm{CsCl}$ structure (Fig. 1). Due to its structural similarity (ignoring the different atom types) to the body-centered-cubic (bcc) Fe structure, we will refer to the $\mathrm{CsCl}$ structure as "bcc-like" throughout this paper, while the metastable $\mathrm{CuAu}$ structure is referred to as "face-centeredcubic-like" ("fcc-like") for the same reason. The AFM state [Fig. 1(a)] is characterized by magnetic moments at the Fe sites of around $3 \mu_{B}$ and negligible moments at the Rh sites, while the FM state [Fig. 1(b)] shows slightly larger moments (around $3.3 \mu_{B}$ ) on the Fe sites and Rh also acquires a moment of about $1 \mu_{B}$ [3]. The phase transition is accompanied by an increase in volume of about $1 \%$ and a large drop in resistivity [2].

In recent years interest in $\mathrm{FeRh}$ has been rejuvenated following its growth in thin-film form, and the associated potential for integration into device architectures. A number of technologically promising behaviors exploiting the coupling between magnetism, resistivity, and volume/strain have been demonstrated. These include magnetic-field writing with resistive reading in $\mathrm{FeRh}$ films grown on $\mathrm{MgO}$ [4], small voltage switching between AFM and FM states for FeRh on ferroelectric $\mathrm{BaTiO}_{3}$ [5], and electric-field phase and resistivity control for FeRh on piezoelectric PMN-PT [6].

A number of density functional theory (DFT) calculations have already been performed to investigate the properties of FeRh. Early work based on the local density approximation (LDA) studied the relative energetics of different magnetic phases and found the ground state to be of type AFM-II (we will refer to this as AFM in the following) [7]. At larger than equilibrium volumes the FM state is energetically preferred within the LDA, which is consistent with the volume increase associated with the AFM to FM phase transition. Subsequent work argued that in analogy to Fe, gradientcorrected functionals (GGA) are required for the correct description of FeRh [8]. It was shown that while LDA and GGA functionals predict similar structures, the magnetic moments on Fe in the AFM phase are significantly larger and in better agreement with experiment using GGA [9]. Later work showed that close to the transition volume, the Fe moments are unstable with respect to canting when treated at the LDA level whereas a semilocal GGA functional stabilizes the collinear FM phase [10]. Using noncollinear LDA calculations it was argued that the Rh moment cannot be described within the Stoner picture as the spin density shows spatial variations around the $\mathrm{Rh}$ atom [11]. This study also established the importance of strong $\mathrm{Fe}-\mathrm{Rh}$ hybridization and showed that the $\mathrm{Fe}-\mathrm{Fe}$ antiferromagnetic interaction is strongly volume dependent, whereas the ferromagnetic $\mathrm{Fe}-\mathrm{Rh}$ interaction remains fairly constant with volume.

Following the recent interest in coherent epitaxial strained FeRh films, some density functional studies have been carried
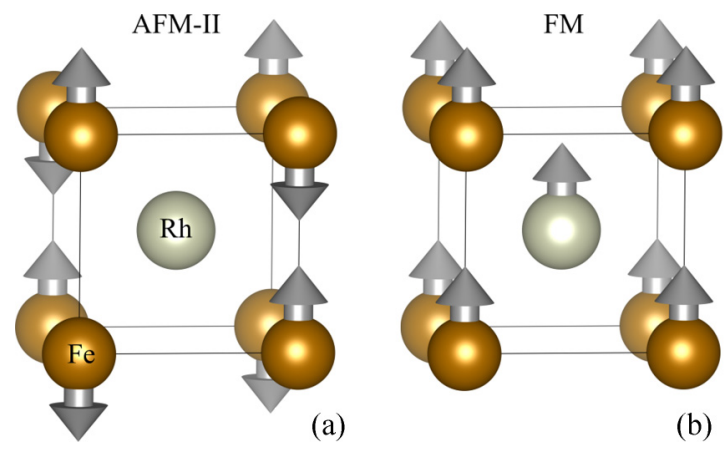

FIG. 1. Magnetic phases of FeRh: (a) antiferromagnetic (AFMII) and (b) ferromagnetic order. 
out for strained systems. In terms of electrical properties, DFT calculations suggested that injecting holes does not significantly affect the relative stability of the AFM and the FM phases, whereas injecting about 0.4 electrons per formula unit makes the FM phase energetically favorable compared to the AFM phase [5]. This was confirmed by calculations of the actual interface between ferroelectric $\mathrm{BaTiO}_{3}$ and $\mathrm{FeRh}$, in which polarized $\mathrm{BaTiO}_{3}$ injects electrons and leads to a favoring of the FM state. The FM magnetic ordering was predicted to have a significantly higher density of states at the Fermi energy, and hence a higher electrical conductivity, than the AFM ordering [5,6,10-12]. No significant change of the density of states at the Fermi energy was found, however, for small $( \pm 0.1 \%)$ strain ranges [6]. It was also shown that for very thin films (9 layers) the FM state is energetically favored over the AFM state [9]. Intriguingly, and relevant for the work that we present here, Ref. [5] showed an unusual energy lowering for the AFM phase under compressive strain at the LDA level, although they did not comment on its existence or its origin.

Here we report results of detailed DFT calculations of the structure of FeRh under strain. We are motivated in part by a lesser known experimental fact about FeRh, namely that FeRh converts to an fcc-like structure when subjected to high velocity impact deformation or other strong deformation such as filing [13,14]. Experimentally this phase transformation was shown to be accompanied by disorder, which might also play a role in the transformation, but which we neglect in the present work as it is not well characterized at this point. The fcc-like structure reverts to the bcc-like FM CsCl structure on heating above $500 \mathrm{~K}$ and finally to the AFM structure when cooling to room temperature, suggesting a Bain path transformation between the bcc-like and fcc-like structures. Indeed, it has been shown using LDA TB-LMTO calculations that the AFM fcc-like state has a lower energy than the FM bcc-like state [15], but a complete investigation of the Bain path in the low-temperature AFM phase is lacking. Understanding the structure and properties of FeRh under both tensile and compressive biaxial strain at $0 \mathrm{~K}$ is an important first step towards modeling the finite-temperature dynamics and hence the metamagnetic transition in coherent epitaxial thin films of FeRh.

Our main finding is that epitaxial strain can convert FeRh from the ground-state AFM bcc-like phase to a metastable AFM fcc-like phase by following the epitaxial Bain path. The behavior manifests in the nonlinear elastic constants as a softening of the orthorhombic shear distortion in the AFM phase under compressive strain. We show that the detailed energetics of the conversion is strongly dependent on the choice of exchange-correlation functional. Using the GGA, which yields better agreement with experiment, the transition from bcc-like to fcc-like structure has a very small energy barrier and the two phases have very similar energies. We predict that the conductivities of the two phases will be very different, based on their different densities of states at the Fermi energy. Finally, our calculations reveal a phonon instability for the AFM bcc-like structure, which we predict will lead to a dimerization of both $\mathrm{Fe}$ and $\mathrm{Rh}$ at low temperature.

\section{COMPUTATIONAL DETAILS}

Our density functional theory calculations were performed using the VASP code [16-19] with both the LDA and PBE [20] density functionals. The influence of correlations was studied using the DFT $+U$ method [21] by applying a rotationally invariant $U$ on the Fe $d$ states [22]. Wave functions were expanded in plane waves up to a kinetic energy cutoff of $550 \mathrm{eV}$. PAW potentials [23,24] with $\mathrm{Fe}(4 s, 3 d, 3 p)$ and $\mathrm{Rh}(5 s, 4 d, 4 p)$ treated as valence electrons were used. Reciprocal space was sampled using a $19 \times 19 \times 19$ gammacentered mesh for the 2 atom unit cell and a $11 \times 11 \times 11$ gamma-centered mesh for the $2 \times 2 \times 216$ atom supercell. These values yield well-converged results for forces, stress, and phonon frequencies. Phonon calculations were performed using the frozen phonon method in the PHONOPY code [25]. Biaxial strain was applied by constraining the length of two of the axes and varying the length of the third axis to find its lowest energy value.

\section{RESULTS AND DISCUSSION}

\section{A. Dependence of properties on the exchange-correlation functional}

Before proceeding to calculate the strain-dependent structure of FeRh, we make an extensive comparison of the properties obtained for the bulk system using different exchangecorrelation functionals, both with literature data from other DFT calculations and with experiment.

\section{Structure and magnetism}

In Fig. 2 we show the energy vs cubic lattice parameter for the nonmagnetic (NM), antiferromagnetic (AFM), and ferromagnetic (FM) phases in the cubic $\mathrm{CsCl}$ structure computed using the LDA [panel (a)] and GGA [panel (b)]. (Note that the NM case is obtained by not allowing spin polarization in the calculation.) Table I compares our optimized lattice parameters and relative energies with results of previously published calculations.

According to experiment, the FM phase has a larger lattice parameter $(2.999 \AA$ A) than the AFM phase $(2.984 \AA$ ) $[3,26]$. We see this also realized in our results-independently of the density functional-as well as in all previous computational

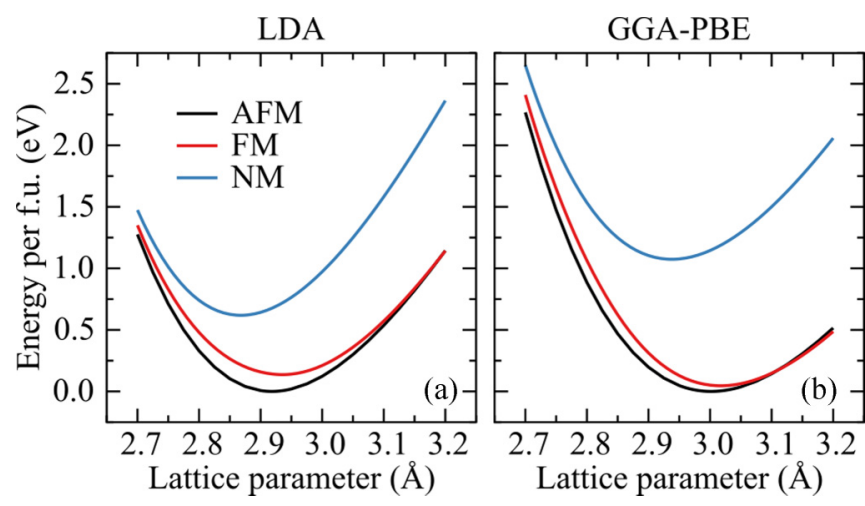

FIG. 2. Energy vs cubic lattice parameter for the nonmagnetic (NM, blue), antiferromagnetic (AFM, black) and ferromagnetic (FM, red) phases of FeRh, computed using (a) the LDA and (b) the GGA. 
TABLE I. Lattice constants, energies per formula unit relative to the AFM phase, and magnetic moments computed in this work and from the literature.

\begin{tabular}{|c|c|c|c|c|c|c|c|}
\hline \multirow[b]{2}{*}{ Phase } & & \multicolumn{3}{|c|}{ LDA } & \multicolumn{3}{|c|}{ GGA } \\
\hline & & $\begin{array}{l}\text { Lattice } \\
(\AA)\end{array}$ & $\begin{array}{l}\text { Energy } \\
(\mathrm{meV})\end{array}$ & $\begin{array}{l}\text { Magn. mom. } \\
\text { Fe, } \mathrm{Rh}\left(\mu_{B}\right)\end{array}$ & $\begin{array}{l}\text { Lattice } \\
\text { (§) }\end{array}$ & $\begin{array}{l}\text { Energy } \\
(\mathrm{meV})\end{array}$ & $\begin{array}{l}\text { Magn. mom. } \\
\mathrm{Fe}, \mathrm{Rh}\left(\mu_{B}\right)\end{array}$ \\
\hline \multirow[t]{7}{*}{ AFM } & This work & 2.918 & & $2.85,0.00$ & 3.002 & & $3.15,0.00$ \\
\hline & Ref. [7] & 2.989 & & $2.98,0.00$ & & & \\
\hline & Ref. [12] & 3.009 & & $3.13,0.00$ & & & \\
\hline & Ref. [8] & & & & 3.002 & & $3.18,0.00$ \\
\hline & Ref. [9] & & & & 2.974 & & $3.11,0.00$ \\
\hline & Ref. [10] & 2.973 & & $3.12,0.00$ & 2.996 & & $3.28,0.00$ \\
\hline & Ref. [5] & 2.922 & & $3.21,0.00$ & & & \\
\hline \multirow[t]{7}{*}{ FM } & This work & 2.935 & 136 & $2.98,1.00$ & 3.018 & 46 & $3.21,1.05$ \\
\hline & Ref. [7] & 3.006 & 52 & $3.15,1.02$ & & & \\
\hline & Ref. [12] & 3.020 & 60 & $3.20,1.02$ & & & \\
\hline & Ref. [8] & & & & 3.020 & 68 & $3.23,1.00$ \\
\hline & Ref. [9] & & & & 2.990 & & $3.19,1.05$ \\
\hline & Ref. [10] & 2.987 & 51 & $3.22,1.04$ & 3.018 & 6 & $3.31,1.02$ \\
\hline & Ref. [5] & 2.935 & 93 & $3.29,0.94$ & & & \\
\hline \multirow[t]{4}{*}{ NM } & This work & 2.868 & 619 & & 2.938 & 1074 & \\
\hline & Ref. [7] & & & & & & \\
\hline & Ref. [12] & 2.958 & 756 & & & & \\
\hline & Ref. [8] & & & & 2.959 & 1088 & \\
\hline
\end{tabular}

studies. Also independently of the functional, the AFM phase is predicted to have a lower energy than the FM phase, a fact that was also reported by all previous DFT studies and is in agreement with the experimental low-temperature AFM ground state. In general the values obtained from our calculations agree with previous reports, the agreement being better for GGA, while our LDA calculations predict around $6 \%$ smaller lattice parameters than previous calculations. We notice, however, that very similar lattice parameters to ours were found by the most recent LDA study [5].

In Table I we also report the predicted magnetic moments. Our computed moments agree well with previously reported values at the LDA [7,10] and GGA [8-10] level, where values of around $3 \mu_{B}$ for the $\mathrm{Fe}$ and $1 \mu_{B}$ for the $\mathrm{Rh}$ moments where reported. These data show that the computed LDA local Fe magnetic moments are about 0.2 to $0.3 \mu_{B}$ smaller than the GGA values for both the AFM and FM phase.

We make three observations at this point: (1) There is a spread of $-6.6 \%$ to $+2.5 \%$ with respect to experiment in the LDA lattice parameters and from $-1.0 \%$ to $+2.1 \%$ in the GGA lattice parameters. A general rule of thumb is that LDA systematically underestimates lattice parameters, while GGA overestimates them. This rule is not observed here, hinting at an extreme sensitivity of the obtained lattice parameters to the density functional and other details of the DFT calculations. (2) The FM phase is predicted to be at least $46 \mathrm{meV}$ per formula unit higher than the AFM phase, except for the GGA calculations of Ref. [10], where an energy difference of only $6 \mathrm{meV}$ was found. Experimentally this energy difference has been determined to be $5.34 \mathrm{meV}$ per formula unit [27], which shows that in general DFT calculations tend to overestimate this energy difference. (3) Under compression, both functionals tend towards a crossover to a nonmagnetic state, indicating the presence of a magnetovolume effect.

\section{Linear elastic constants}

To further assess the performance of different density functionals, we report in Table II the elastic constants computed by fitting to isostatic as well as volume-conserving orthorhombic and tetragonal strain in a range of $-5 \%$ to $+5 \%$ [28]. We show values for the FM and AFM phases, as well as NM for completeness. For the AFM phase at the LDA level, the range had to be reduced to $-2.5 \%$ to $+2.5 \%$ in order to obtain a good fit in the orthorhombic strain case, hinting already that these second-order elastic constants do not fully capture the elastic behavior of FeRh and that we might anticipate unusual strain-dependent behavior.

TABLE II. Elastic constants (in GPa) for the different magnetic phases of FeRh computed using the LDA and GGA-PBE functionals.

\begin{tabular}{lcrr}
\hline \hline Phase & & LDA & GGA-PBE \\
\hline AFM & $B$ & 246.802 & 195.978 \\
& $C_{11}-C_{12}$ & -12.850 & 44.206 \\
& $C_{11}$ & 238.236 & 225.449 \\
& $C_{12}$ & 251.086 & 181.243 \\
& $C_{44}$ & 132.891 & 119.867 \\
FM & $B$ & 213.441 & 193.298 \\
& $C_{11}-C_{12}$ & 66.626 & 87.397 \\
& $C_{11}$ & 257.858 & 251.563 \\
& $C_{12}$ & 191.232 & 164.166 \\
& $C_{44}$ & 118.751 & 109.773 \\
NM & $B$ & 295.075 & 239.574 \\
& $C_{11}-C_{12}$ & -314.345 & -262.455 \\
& $C_{11}$ & 85.512 & 64.604 \\
& $C_{12}$ & 399.857 & 327.059 \\
& $C_{44}$ & 166.448 & 137.492 \\
\hline \hline
\end{tabular}


Experimentally, the AFM phase (ground state in a $\mathrm{Fe}_{0.98} \mathrm{Rh}_{1.02}$ alloy) was measured to have a slightly higher bulk modulus of $141 \mathrm{GPa}$ than the FM phase (ground state in $\mathrm{Fe}_{1.04} \mathrm{Rh}_{0.96}$ ) with $133 \mathrm{GPa}$ [29]. Despite the slightly different composition, we expect the elastic constants in $\mathrm{Fe}_{1.00} \mathrm{Rh}_{1.00}$ to be comparable. Our predicted bulk moduli are higher than these experimental values, especially at the LDA level, where the values are overestimated by a factor of 1.60 and 1.74 respectively for the AFM and FM phases. The GGA predictions are closer to experiment but still overestimate by a factor 1.36 and 1.45 , respectively. Despite this overestimation, it is encouraging that the AFM phase is predicted to have a higher bulk modulus than the FM phase. Our results are also consistent with previous predictions at the LDA level (AFM: 214.4 GPa [7], 226.8 GPa [12], $245.4 \mathrm{GPa}$ [10]; FM: 201.6 GPa [7], 244.0 GPa [12], 236.4 GPa [10]) as well as the GGA level (AFM: $197 \mathrm{GPa}$ [8], $219.4 \mathrm{GPa}$ [10]; FM: $193 \mathrm{GPa}$ [8], 218.1 GPa [10]).

The data in Table II have two particularly interesting implications, given that a material is elastically stable only when $C_{11}-C_{12}>0$. First, we see that, independently of the functional, the NM phase does not satisfy this criterion and is hence elastically unstable, implying that the formation of local moments and their interaction is required to structurally stabilize FeRh. It is somewhat surprising, however, that the AFM phase is also elastically unstable when using the LDA functional whereas it is stable using GGA. This incomplete stabilization could be attributed to the smaller magnetic moments, which yield reduced magnetic interactions at the LDA level. The FM phase on the other hand is elastically stable independently of the functional.

We conclude at this point that based on structural parameters, elastic properties, and size of the magnetic moments, the GGA yields a better description of bulk FeRh than the LDA. In what follows, therefore, we use PBE-GGA exclusively for calculating the strain-dependent structure. Since we focus on the ground-state AFM ordering, the overestimate of the energy difference to the FM phase, which is one inherent problem of the PBE functional, will not affect our conclusions, but we emphasize that care should be taken when considering the relative stability of the different magnetic orderings using DFT calculations.

\section{B. Epitaxial strain dependence}

In Fig. 3(a) we show the energy evolution of the AFM phase as a function of epitaxial strain for the PBE functional and for the sake of completeness also for the LDA functional. While under tensile strain both functionals predict the expected approximately quadratic increase in energy with increasing strain, the functionals behave markedly differently under compressive strain.

Using LDA [Fig. 3(b)], the bcc-like $\mathrm{CsCl}$ structure of the AFM phase is merely an inflection point in the energy landscape and we observe a significant energy lowering under compressive epitaxial strain, which is a manifestation of the negative $C_{11}-C_{12}$ value reported in Table II. A similar energy evolution was previously shown but not commented upon [5]. Accompanying the reduction in energy is an increase in the $c / a$ ratio [Fig. 3(b)] towards $\sqrt{2}=1.414$, indicating that a

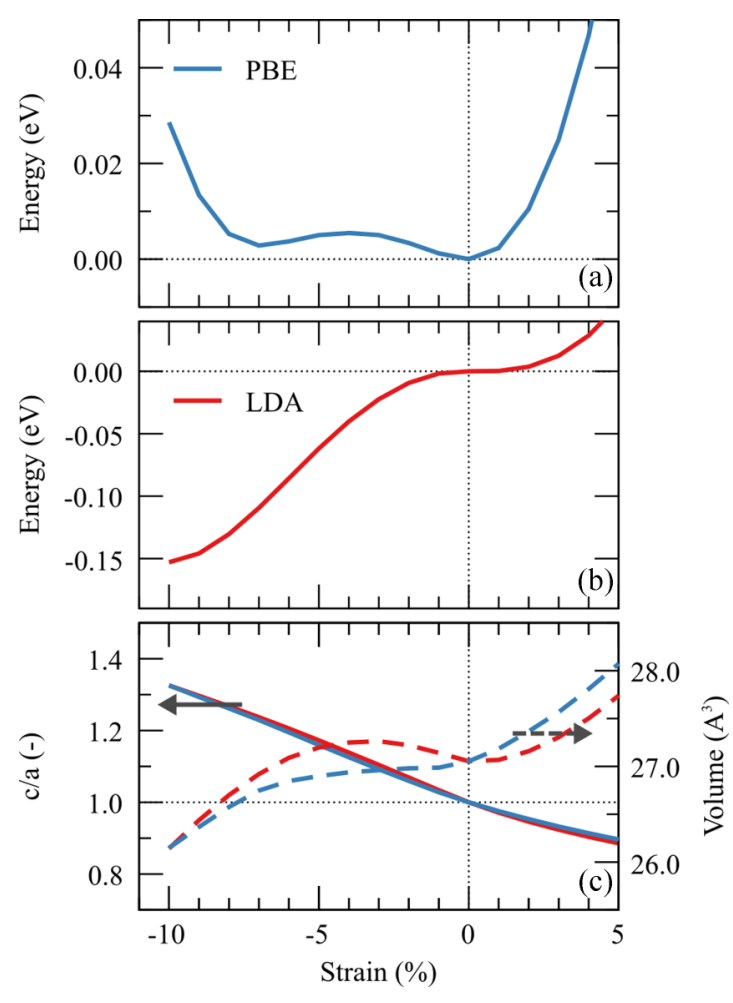

FIG. 3. Calculated strain dependence of the energy of the AFM phase computed at (a) the GGA-PBE and (b) the LDA level along with (c) their respective $c / a$ ratios (solid lines, left axis) and volume (dashed lines, right axis).

transition along the tetragonal Bain path has taken FeRh from the bcc-like $\mathrm{CsCl}$ structure to an fcc-like structure.

At the GGA level [Fig. 3(a)], which we concluded above yields a better description of FeRh, the energy of the AFM phase increases under compressive strain and the bcc-like $\mathrm{CsCl}$ structure is predicted to be the ground state. There exists however a metastable state at $-7 \%$ strain, only $3 \mathrm{meV} /$ f.u. higher in energy than the bcc-like $\mathrm{CsCl}$ structure and reached from the bcc-like phase by overcoming a small energy barrier of $5 \mathrm{meV} / \mathrm{f}$.u. This is in agreement with the experimental finding that plastic deformation can induce a transformation from bcc-like to fcc-like, which is reversed upon heating $[13,14]$.

Another implication of this result is that FeRh should exhibit a very nonstandard behavior under compressive strain. At $0 \%$ strain and around 7\% strain, one should consider the material unstrained, but existing in two distinct phases (bcc-like and fcc-like, respectively). If grown at intermediate strains, the material could relax into either of the two phases as the film grows thicker, which could lead to phase coexistence in partially relaxed films, reminiscent of the different phases occurring in $\mathrm{BiFeO}_{3}$ [30]. Figure 3(c) reveals that the volume change is minimal for the strain range encompassing the two energy minima, further supporting a phase coexistence scenario.

In Fig. 4 we show the GGA-computed electronic densities of states (DOSs) for the bcc-like (a) FM and (b) AFM phases, as well as (c) the metastable fcc-like AFM structure at 7\% compressive strain. All DOSs are reported per formula unit 


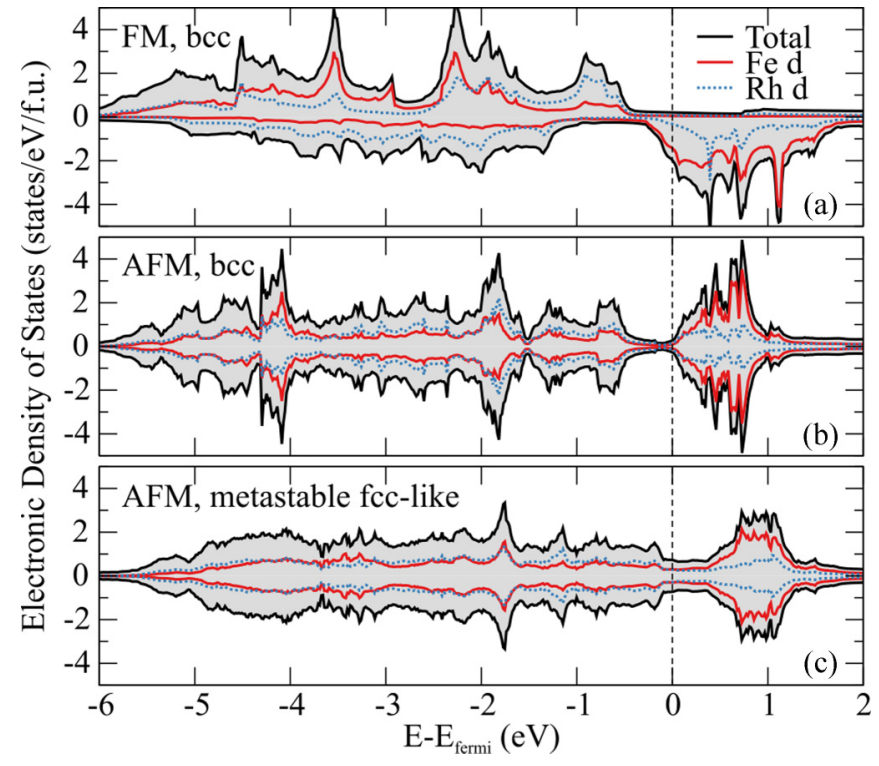

FIG. 4. Electronic densities of states (DOS) for (a) the bcc-like FM, (b) the bcc-like AFM, and (c) the metastable fcc-like AFM phases.

of FeRh so that the DOS at the Fermi energy can be directly compared for the different structures. The much larger DOS at the Fermi energy for the bcc-like FM phase compared to the bcc-like AFM phase is in agreement with previous calculations [8-10]. Comparing the DOS for the metastable fcc-like AFM structure [Fig. 4(c)], it is interesting to note that this structure has almost as large a DOS at the Fermi energy as the FM phase, when considering both up and down spin electrons. An enhancement of the DOS at the Fermi energy was previously predicted to occur in the FM phase under compressive strain [15]. Under the assumption that other conductivity-determining parameters such as the Fermi velocity and the carrier lifetime do not change significantly between the different phases, we thus predict that a change from the AFM bcc-like to the AFM fcc-like structure will result in a change in resistivity comparable to that accompanying the AFM-FM phase transition. During the review process a comprehensive study on the conductivity in AFM and FM FeRh was posted, to which we refer the reader [31].

In addition to this elastic instability that manifests under compressive strain, our calculations reveal a lattice instability corresponding to dimerizations of $\mathrm{Fe}$ and $\mathrm{Rh}$ atoms along perpendicular directions. In Fig. 5(a) we show the evolution of the phonon spectrum in the AFM phase, computed using GGA-PBE, as a function of strain. It can be seen that at $0 \%$ strain a lattice instability exists at the degenerate $\mathrm{M}$ and $\mathrm{R}$ points that manifests as an imaginary phonon frequency. Compressive strain lifts the degeneracy of the $\mathrm{M}$ and $\mathrm{R}$ points and rapidly suppresses this instability at the $\mathrm{M}$ point [wave vector in the compressively strained plane; see Fig. 5(c)], while it is enhanced at the $\mathrm{R}$ point (wave vector with a component along the elongated out-of-plane axis). In Fig. 5(b) we show the evolution of this R-point frequency with strain. The instability is initially enhanced up to compressive strains of $3 \%$ to $4 \%$ and is then rapidly suppressed and vanishes for compressive strain slightly below $7 \%$.
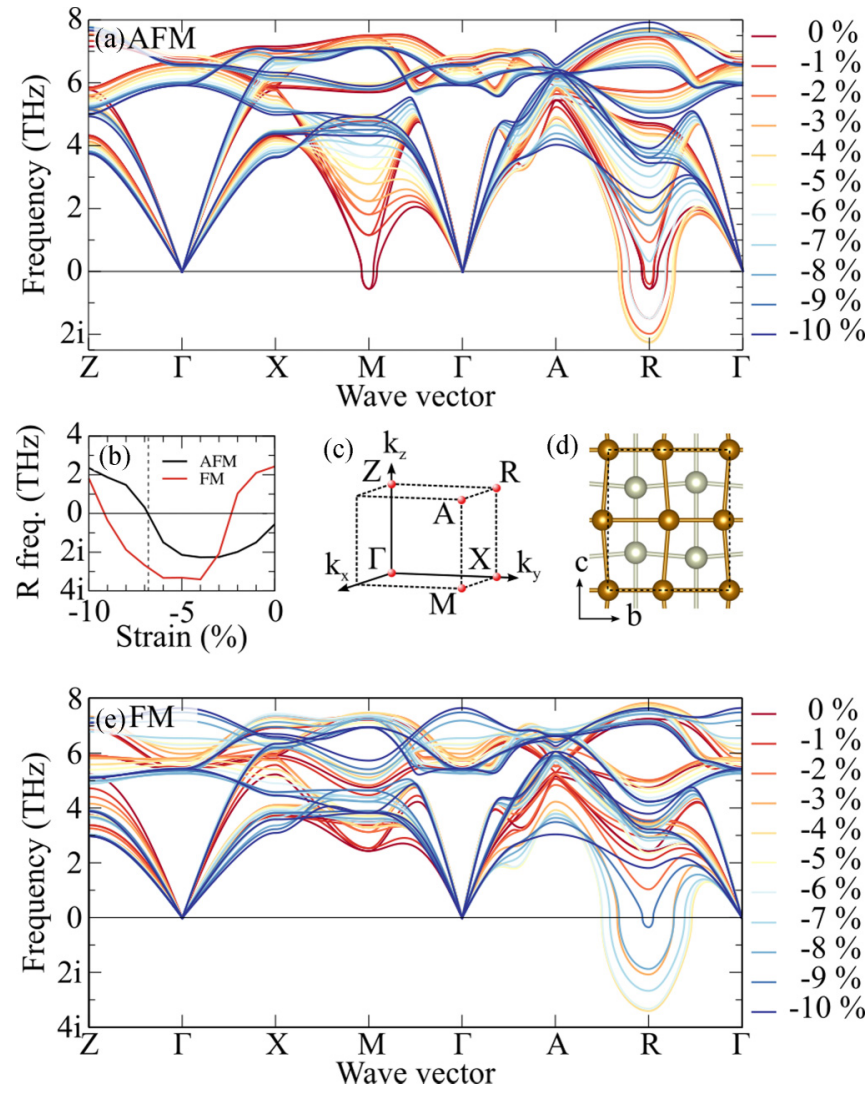

FIG. 5. (a) Compressive strain dependence of the phonon dispersion in AFM FeRh computed at the GGA level. In (b) we show the evolution of the frequency as a function of strain at the unstable R point. In (c) and (d) we show the high-symmetry points in the tetragonal Brillouin zone and the resulting structural distortion, respectively. Panel (e) shows the evolution of the phonon dispersion in FM FeRh as a function of compressive strain.

The amounts of strain where the instability is maximal as well as where it vanishes are in surprisingly good agreement with the position of the top of the energy barrier and the metastable minimum, respectively, of the energy curve in Fig. 3(a). It is known that Bain path transitions can be linked to unstable phonon modes [32]; however in the present case, the instability appears only in a small portion of $q$ space around the $\mathrm{R}$ point. Such zone-boundary instabilities cannot be invoked to explain a Bain path transition, which relies on unstable long-wave-length phonons [32]. We have verified that no long-wave-length instability exists along the R- $\Gamma$ line, which could mean that the strain match might be a coincidence. Nevertheless it is interesting to note that both the present R-point instability as well as an elastic $C^{\prime}$ instability are represented by transverse [110] modes.

In Fig. 5(d) we show the structural distortion associated with this instability condensed at its most energy-lowering amplitude for the 5\% compressively strained structure. We see that it corresponds to a dimerization of the $\mathrm{Rh}$ atoms along the film normal and a dimerization of the $\mathrm{Fe}$ atoms in the strain plane. The distortion results in an energy lowering of $10.8 \mathrm{meV}$ per FeRh formula unit at this strain value. In the unstrained cubic bcc-like structure the energy lowering is about two orders of magnitude smaller at only $0.125 \mathrm{meV}$ 
per formula unit. This small energy scale suggests that the occurrence of this phenomenon should be limited to very low temperatures, consistent with this structure not yet having been observed in experiment. We hope that our prediction will motivate such studies in the future. Compressive strain could however trigger the dimerization at higher temperatures. In terms of electrical properties, the unstable phonon has the effect of slightly lowering the DOS at the Fermi energy, at $5 \%$ compressive strain going from 0.6 states $\mathrm{eV}^{-1}$ f.u. ${ }^{-1}$ in the unmodulated structure to 0.5 states $\mathrm{eV}^{-1}$ f.u. ${ }^{-1}$ in the modulated structure.

In Fig. 5(e) we show the phonon dispersion curve for the FM phase as a function of strain. Comparing it to the AFM case shown in Fig. 5(a), we can see that the FM phase does not have a phonon instability at the $\mathrm{M}$ and $\mathrm{R}$ points at $0 \%$ strain as opposed to the AFM phase. The instability can however be triggered by applying compressive strain larger than $2 \%$. This difference between the AFM and the FM phases is shown more clearly by the evolution of the R-point frequencies depicted in Fig. 5(b), where we see that the FM phase has a real frequency at $0 \%$ strain before developing the instability for compressive strain between $2 \%$ and $9 \%$. The appearance of the instability is most likely caused by the reduction of the magnitude of the local moments with applied strain. The observed lattice instability is consistent with the elastic behavior, where the FM phase was further from an instability than the AFM phase, indicating that stronger magnetic interactions driven by the larger local moments in the FM phase play a crucial role in stabilizing the FeRh structure.

Finally for this section, we present results of computer experiments to test the role of the size of the magnetic moments in determining the stability of the AFM phase with respect to epitaxial tetragonal strain. In order to check whether a further increase of the moments would lead to a complete stabilization of the AFM phase, we apply an on-site DFT $+U$ correction to the $\mathrm{Fe} d$ orbitals. We strongly emphasize that we do not propose this as a good physical description of FeRh, but use it as a computational trick to tune the magnitude of the magnetic moments by modifying the charge localization on the $\mathrm{Fe}$ atoms. We also note that by using this computational trick, we change both the magnitude of the local moments and the localization on the Fe $d$ orbitals; in fact we directly control the latter with the $U$ parameter and this in turn affects the former. Constrained-moment calculations as an alternative method would directly affect the magnitude of the moment, which results in changes in charge localization, thus also probing both effects simultaneously. In Fig. 6(a), we show the evolution of the energy as a function of strain for different values of $U$ added to a GGA calculation. As shown above, without this artificial increase of the moments $(U=0.00 \mathrm{eV})$, the secondary minimum of slightly higher energy exists for compressive strain. Upon increasing $U$, the secondary minimum disappears around $U \sim 0.25 \mathrm{eV}$, but remains visible as a kink in the energy curve. As is to be expected, increasing $U$ increases the magnitude of the magnetic moments as shown in Fig. 6(b). Going from $U=0 \mathrm{eV}$ to $1 \mathrm{eV}$, increases the moments by $0.2 \mu_{B}$ and more. This confirms indeed that increasing the magnetic moments will completely stabilize the bcc-like $\mathrm{CsCl}$ structure with respect to tetragonal strain. These results also imply, however, that the magnetic moment magnitudes obtained from a pure GGA calculation are required to achieve
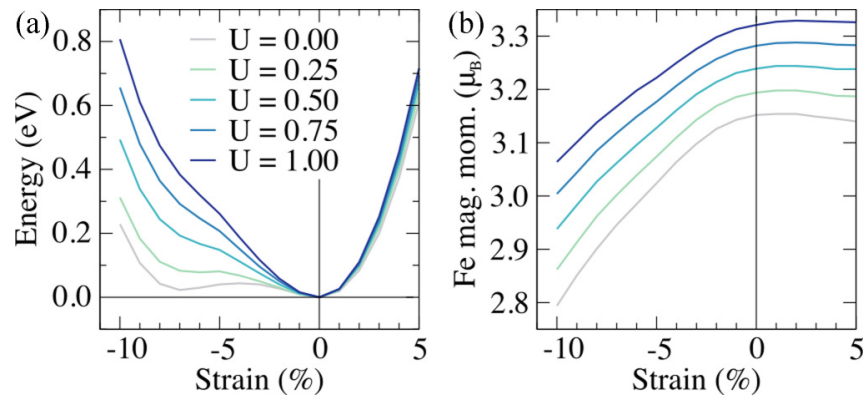

FIG. 6. (a) GGA $+U$ relative energy of the AFM phase with $U$ values between 0 and $1 \mathrm{eV}$ and (b) the corresponding magnetic moments.

agreement with the experimentally observed transformation to a metastable fcc-like phase. Artificially increasing the magnetic moments, for example with the DFT $+U$ method, does not yield a physically relevant description of FeRh.

Our findings also confirm our assertion that the addition of a Hubbard- $U$ correction is not a physically appropriate description. As we mentioned in the introduction, experimental evidence supports the existence of an fcc-like phase, which can be reached by high energetic deformations of the material $[13,14]$. However, addition of a $U>0.25$ to the GGA functional suppresses this state completely. This leads us to believe that semilocal functionals without any on-site correction are the best choice to describe the intermetallic compound $\mathrm{FeRh}$ in a density functional theory calculation.

\section{Nonlinear elasticity}

Finally we analyze the anomalous elastic behavior of the AFM state under compressive epitaxial strain seen in Fig. 3(a) in the framework of nonlinear elasticity. In particular, we investigate how the linear elastic constant $C^{\prime}=\left(C_{11}-C_{12}\right) / 2$ varies with strain, and which orders in the energy expansion we need to include in order to give a good description of the equilibrium AFM state and the surrounding saddle-point region. Our main findings are first an unusually strong dependence of the linear elastic constants with strain, with $C^{\prime}$ undergoing a strong softening under compression, and second that elastic constants up to fifth order are required to describe the elastic energy surface. These results confirm that the ground state of $\mathrm{FeRh}$ is proximal to an elastic instability.

Using the GGA exchange-correlation functional that we established above to give the best description of FeRh, we performed two series of deformations to extract the elastic constants: first an isotropic volume distortion followed by either a volume-conserving monoclinic or orthorhombic distortion [28]. This allowed us to express the tension/compression asymmetry, which is normally fitted to a Murnaghan-Birch fit $[33,34]$, directly in terms of the higher-order elastic constants:

$$
\begin{aligned}
E= & \eta^{2}\left(\frac{3 C_{11}}{2}+3 C_{12}\right)+\eta^{3}\left(\frac{C_{111}}{2}+3 C_{112}+C_{123}\right) \\
& +\eta^{4}\left(\frac{C_{1111}}{8}+C_{1112}+\frac{3 C_{1122}}{4}+\frac{3 C_{1123}}{2}\right) \\
& +\eta^{5}\left(\frac{C_{11111}}{40}+\frac{C_{11112}}{4}+\frac{C_{11122}}{2}+\frac{C_{11123}}{2}+\frac{3 C_{11223}}{4}\right),
\end{aligned}
$$


TABLE III. AFM FeRh elastic constants obtained by fitting to DFT calculations of both orthorhombic and monoclinic distortions as a function of an initial isotropic distortion, and epitaxial-strain distortions. Slight deviations of the linear coefficients with respect to the values in Table II stem from the different formalism and fitting procedure.

\begin{tabular}{lc}
\hline \hline Elastic constants & AFM $(\mathrm{GPa})$ \\
\hline$C_{11}, C_{12}, C_{44}$ & $224.57,184.60,128.05$ \\
$C_{111}, C_{112}, C_{123}$ & $-2380.67,-561.34,-1461.22$ \\
$C_{144}, C_{166}, C_{456}$ & $56424.12,-29201.20,-$ \\
$C_{1111}, C_{1112}$ & $27774.20,646.69$ \\
$C_{1122}, C_{1123}$ & $1206.44,6456.56$ \\
$C_{1144}, C_{1155}$ & $5875091.80,-2355521.45$ \\
$C_{1255}, C_{1266}$ & $647062.69,-1860996.55$ \\
$C_{1456}, C_{4444}$ &,--71474.99 \\
$C_{4455}$ & - \\
$C_{11111}, C_{11112}$ & $-444308.22,-22012.63$ \\
$C_{11122}, C_{11123}$ & $39249.66,60554.46$ \\
$C_{11144}, C_{11155}$ & $252799931.07,-189779777.14$ \\
$C_{11223}, C_{11244}$ & $-79195.94,-10605443.05$ \\
$C_{11255}, C_{11266}$ & $87944526.61,61761708.62$ \\
$C_{11456}, C_{11244}$ &,--10605443.05 \\
$C_{12456}, C_{14444}$ &,--501845412.25 \\
$C_{14455}, C_{15555}$ &,- 253346458.03 \\
$C_{15566}, C_{44456}$ &,-- \\
\hline \hline
\end{tabular}

where $\eta$ is the diagonal term of the Lagrangian strain matrix associated with isotropic expansion (for a review of nonlinear elasticity theory see the Appendix). Second, we performed an isotropic in-plane distortion $\left(\varepsilon_{1}\right)$ plus a perpendicular out-ofplane distortion $\left(\varepsilon_{2}\right)$, corresponding to a biaxial strain state. We then fitted the nonlinear elastic energy expression, Eq. (A5), to our DFT database of energies versus distortions using a simulated annealing algorithm [35]. The resulting optimal numerical values of the nonlinear elastic constants are listed in Table III. Note that the chosen distortions do not directly probe all elastic constants. For example $C_{456}, C_{1456}, C_{4455}$, $C_{11456}, C_{12456}, C_{14455}, C_{15566}$, and $C_{44456}$ do not contribute to the elastic energies and are therefore omitted from the fitting process, and others enter only in linear combinations [for example, see Eqs. (1) and (2)]. Thus caution should be taken in interpreting the numerical values of individual higher-order elastic constants. For conciseness we present results only for the AFM phase.

Figure 7(a) shows the change in elastic energy as a function of the volume-conserving orthorhombic distortion for different isotropic strains. Both the DFT data and the nonlinear elasticity fit are shown, where the zero energy (at zero orthorhombic strain) is set to the AFM energy per formula unit of the given isotropic strain. We see that the $C^{\prime}$ shear modulus changes as a function of isotropic strain (as does the $C_{44}$ shear modulusnot shown). In particular there is a strong softening of the $C^{\prime}$ modulus at negative isotropic strains (corresponding to lattice contraction). For the FM phase, no softening of the FM $C^{\prime}$ modulus under isotropic compression was seen and for the NM phase, the $C^{\prime}$ modulus was always negative indicating a broad regime of elastic instability as suggested in Sec. III A 2.

Insight into the origin of this behavior can be gained by writing down the isotropic strain dependence of the $C^{\prime}$ elastic
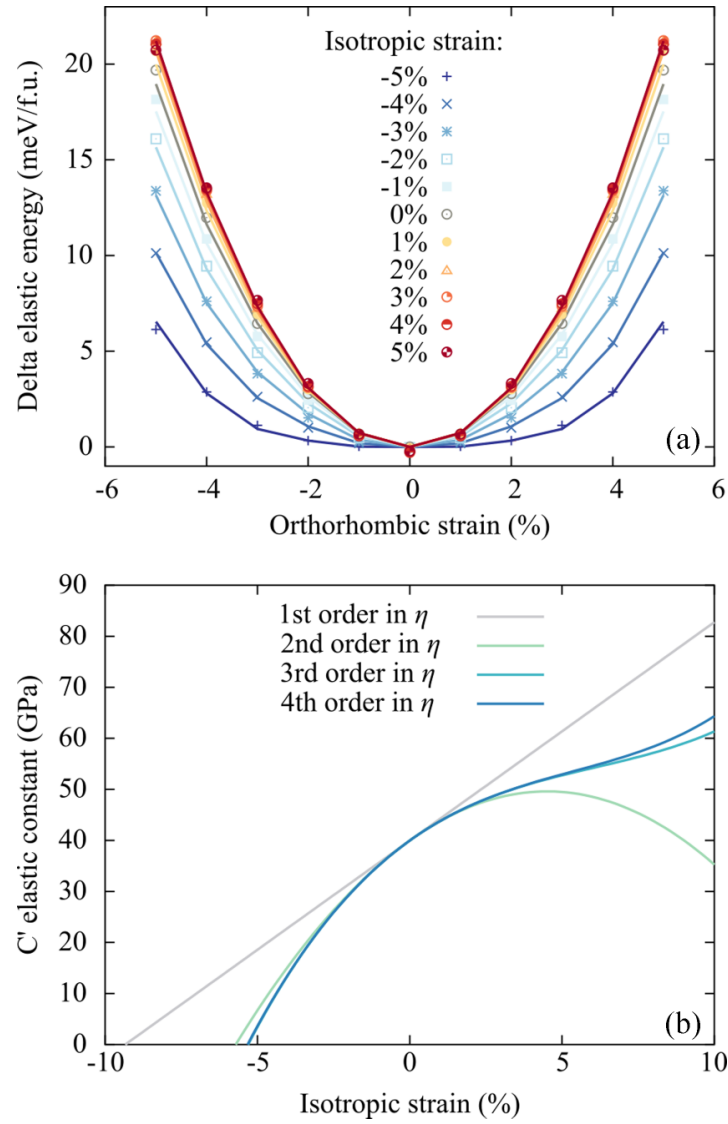

FIG. 7. (a) Change in elastic energy (per formula unit) at a finite isotropic strain with respect to a volume-conserving orthorhombic strain for the AFM FeRh system. For each color the points show the DFT data and the lines the Eq. (A5) fit for a specific value of isotropic strain. (b) Dependence of the AFM shear moduli $C^{\prime}$ on isotropic strain according to Eq. (2). Here different orders with respect to the isotropic strain are shown. Convergence under compression is achieved at fourth order.

constant in terms of the higher-order elastic constants. Up to quadratic order with respect to the isotropic strain, $\eta$, this is

$$
\begin{aligned}
C^{\prime}(\eta)= & \frac{1}{2}\left(C_{11}-C_{12}\right)+\frac{1}{2}\left(6 C_{11}+C_{111}-C_{123}\right) \eta \\
& +\frac{1}{2}\left(11 C_{11}+4 C_{12}+\frac{11 C_{111}}{2}+6 C_{112}\right. \\
& \left.-\frac{5 C_{123}}{2}+\frac{C_{1111}}{2}+C_{1112}-\frac{3 C_{1123}}{2}\right) \eta^{2}+\cdots .
\end{aligned}
$$

A similar expression for the $C_{44}$ constant can also be obtained. Inspection of Eq. (2) reveals that the higher-order elastic constants strongly affect each coefficient of the $\eta$ expansion. Not shown are the $\eta^{3}$ and $\eta^{4}$ terms, which include respectively the fourth and fifth order order elastic constants. In Fig. 7(b) we plot $C^{\prime}$ including contributions up to fourth order in $\eta$ using the obtained numerical elastic constants for the AFM state for a range of negative and positive isotropic strains. We see again that, consistent with Fig. 7(a), $C^{\prime}$ softens on lattice contraction, eventually becoming negative at an isotropic 
strain of approximately $-5 \%$. Thus under a sufficiently large isotropic compression the AFM state becomes unstable to an orthorhombic distortion. For the considered range of isotropic compressions, this trend is not seen in the FM phase.

Finally we note that the elastic constant behavior of FeRh is remarkably similar to that of pure bcc-like Fe, which also exhibits a $C^{\prime}$ softening to zero under compression, and has a negative $C^{\prime}$ for the NM phase. For bcc-like Fe, this phenomenon is associated with the suppression of itinerant magnetism under compression, and it has been pointed out that this is a manifestation of the magnetovolume effect [36-38]. As we pointed out earlier (Fig. 2), the energies of the AFM, FM, and NM phases tend to the same values for sufficiently high compressions, and this is accompanied by a reduction in the size of the magnetic moments. For example, at an isotropic compression of $5 \%$, the individual Fe moment values of the AFM FeRh phase are lower than their equilibrium values by approximately $10 \%$.

\section{CONCLUSIONS}

In conclusion, we have shown that the GGA exchangecorrelation functional provides a reasonable description of the known structural properties of FeRh, in contrast to the LDA which predicts incorrectly that a competing fcc-like phase with smaller lattice constant is the ground state, and the GGA $+U$ method which incorrectly completely destabilizes this competing phase. At the GGA level the fcc-like state is metastable, consistent with reports of its existence under high-impact deformation, and we predict that it can be reached with compressive epitaxial strain. Due to an enhanced density of states at the Fermi energy, this fcc-like state shows a decreased electrical resistivity compared to the bcc-like AFM phase. We rationalize the behavior using nonlinear elasticity theory, and predict the existence of a lattice instability, which should manifest at low temperatures as a dimerization of both $\mathrm{Fe}$ and $\mathrm{Rh}$ atoms along perpendicular directions.

\section{ACKNOWLEDGMENTS}

This work was financially supported by the ETH Zürich and by the ERC Advanced Grant program, No. 291151. This work was supported by a grant from the Swiss National Supercomputing Centre (CSCS) under project ID s624.

\section{APPENDIX: NONLINEAR ELASTICITY: DEFINITIONS AND FITTING PROCEDURE}

Nonlinear elasticity expands the elastic energy to supralinear order with respect to the deformation matrix, and thus defines higher than linear order elastic coefficients. Unlike linear elasticity, nonlinear elasticity involves finite distortions of a material and therefore allows for the study of $C^{\prime}$ and $C_{44}$ as a function of an arbitrary distortion. Here we outline nonlinear elasticity theory to fifth order.

The higher-order elastic constants depend on the precise definition of strain. Here the Lagrangian strain is used, which gives a measure of the finite displacement of a material point [39-41]. The Lagrangian strain is formally defined as

$$
\boldsymbol{\eta}=\frac{1}{2}\left(\mathbf{J J}^{T}-\mathbf{1}\right)
$$

where the Jacobian matrix, $\mathbf{J}$, is obtained from the linear strain tensor,

$$
\mathbf{J}=\mathbf{1}+\boldsymbol{\varepsilon}
$$

Thus the Lagrangian strain is

$$
\boldsymbol{\eta}=\boldsymbol{\varepsilon}+\frac{1}{2} \boldsymbol{\varepsilon}^{2} .
$$

In terms of Voigt notation, the most general elastic energy density is written as

$$
E=\frac{1}{2 !} \sum_{i j} C_{i j} \eta_{i} \eta_{j}+\frac{1}{3 !} \sum_{i j k} C_{i j k} \eta_{i} \eta_{j} \eta_{k}+\cdots .
$$

Here $\eta_{1}=\eta_{11}, \eta_{2}=\eta_{22}, \eta_{3}=\eta_{33}, \eta_{4}=2 \eta_{23}, \eta_{5}=2 \eta_{13}$, and $\eta_{6}=2 \eta_{12}$. To fifth order, the elastic energy density becomes

$$
E=\phi_{2}+\phi_{3}+\phi_{4}+\phi_{5}+\cdots,
$$

where for a cubic system the linear elastic contribution is given by

$$
\begin{aligned}
\phi_{2}= & \frac{C_{11}}{2}\left(\eta_{1}^{2}+\eta_{2}^{2}+\eta_{3}^{2}\right)+\frac{C_{44}}{2}\left(\eta_{4}^{2}+\eta_{5}^{2}+\eta_{6}^{2}\right) \\
& +C_{12}\left(\eta_{1} \eta_{2}+\eta_{3} \eta_{2}+\eta_{1} \eta_{3}\right) .
\end{aligned}
$$

The cubic higher-order nonlinear elastic energy terms are

$$
\begin{aligned}
\phi_{3}= & \frac{C_{111}}{6}\left(\eta_{1}^{3}+\eta_{2}^{3}+\eta_{3}^{3}\right)+\frac{C_{112}}{2}\left(\eta_{2} \eta_{1}^{2}+\eta_{3} \eta_{1}^{2}+\eta_{2}^{2} \eta_{1}+\eta_{3}^{2} \eta_{1}+\eta_{2} \eta_{3}^{2}+\eta_{2}^{2} \eta_{3}\right)+C_{123} \eta_{1} \eta_{2} \eta_{3} \\
& +\frac{C_{144}}{2}\left(\eta_{1} \eta_{4}^{2}+\eta_{2} \eta_{5}^{2}+\eta_{3} \eta_{6}^{2}\right)+\frac{C_{166}}{2}\left(\eta_{2} \eta_{4}^{2}+\eta_{3} \eta_{4}^{2}+\eta_{1} \eta_{5}^{2}+\eta_{3} \eta_{5}^{2}+\eta_{1} \eta_{6}^{2}+\eta_{2} \eta_{6}^{2}\right)+C_{456} \eta_{4} \eta_{5} \eta_{6} \\
\phi_{4}= & \frac{C_{1111}}{24}\left(\eta_{1}^{4}+\eta_{2}^{4}+\eta_{3}^{4}\right)+\frac{C_{1112}}{6}\left[\eta_{1}^{3}\left(\eta_{2}+\eta_{3}\right)+\eta_{2}^{3}\left(\eta_{3}+\eta_{1}\right)+\eta_{3}^{3}\left(\eta_{1}+\eta_{2}\right)\right] \\
& +\frac{C_{1122}}{4}\left(\eta_{1}^{2} \eta_{2}^{2}+\eta_{2}^{2} \eta_{3}^{2}+\eta_{3}^{2} \eta_{1}^{2}\right)+\frac{C_{1123}}{2}\left(\eta_{1}^{2} \eta_{2} \eta_{3}+\eta_{2}^{2} \eta_{3} \eta_{1}+\eta_{3}^{2} \eta_{1} \eta_{2}\right) \\
& +\frac{C_{1144}}{4}\left(\eta_{1}^{2} \eta_{4}^{2}+\eta_{2}^{2} \eta_{5}^{2}+\eta_{3}^{2} \eta_{6}^{2}\right)+\frac{C_{1155}}{4}\left[\eta_{1}^{2}\left(\eta_{5}^{2}+\eta_{6}^{2}\right)+\eta_{2}^{2}\left(\eta_{4}^{2}+\eta_{6}^{2}\right)+\eta_{3}^{2}\left(\eta_{4}^{2}+\eta_{5}^{2}\right)\right] \\
& +\frac{C_{1255}}{2}\left[\eta_{1} \eta_{2}\left(\eta_{4}^{2}+\eta_{5}^{2}\right)+\eta_{2} \eta_{3}\left(\eta_{5}^{2}+\eta_{6}^{2}\right)+\eta_{1} \eta_{3}\left(\eta_{4}^{2}+\eta_{6}^{2}\right)\right]+\frac{C_{1266}}{2}\left(\eta_{1} \eta_{2} \eta_{6}^{2}+\eta_{2} \eta_{3} \eta_{4}^{2}+\eta_{1} \eta_{3} \eta_{5}^{2}\right) \\
& +C_{1456} \eta_{4} \eta_{5} \eta_{6}\left(\eta_{1}+\eta_{2}+\eta_{3}\right)+\frac{C_{4444}}{24}\left(\eta_{4}^{4}+\eta_{5}^{4}+\eta_{6}^{4}\right)+\frac{C_{4455}}{4}\left(\eta_{4}^{2} \eta_{5}^{2}+\eta_{5}^{2} \eta_{6}^{2}+\eta_{6}^{2} \eta_{4}^{2}\right)
\end{aligned}
$$




$$
\begin{aligned}
\phi_{5}= & \frac{C_{11111}}{120}\left(\eta_{1}^{5}+\eta_{2}^{5}+\eta_{3}^{5}\right)+\frac{C_{11112}}{24}\left[\eta_{1}^{4}\left(\eta_{2}+\eta_{3}\right)+\eta_{2}^{4}\left(\eta_{1}+\eta_{3}\right)+\eta_{3}^{4}\left(\eta_{1}+\eta_{2}\right)\right] \\
& +\frac{C_{11122}}{12}\left[\eta_{1}^{3}\left(\eta_{2}^{2}+\eta_{3}^{2}\right)+\eta_{2}^{3}\left(\eta_{1}^{2}+\eta_{3}^{2}\right)+\eta_{3}^{3}\left(\eta_{1}^{2}+\eta_{2}^{2}\right)\right]+\frac{C_{11123}}{6}\left(\eta_{1}^{3} \eta_{2} \eta_{3}+\eta_{2}^{3} \eta_{1} \eta_{3}+\eta_{3}^{3} \eta_{1} \eta_{2}\right) \\
& +\frac{C_{11144}}{12}\left(\eta_{1}^{3} \eta_{4}^{2}+\eta_{2}^{3} \eta_{5}^{2}+\eta_{3}^{3} \eta_{6}^{2}\right)+\frac{C_{11155}}{12}\left[\eta_{1}^{3}\left(\eta_{5}^{2}+\eta_{6}^{2}\right)+\eta_{2}^{3}\left(\eta_{4}^{2}+\eta_{6}^{2}\right)+\eta_{3}^{3}\left(\eta_{4}^{2}+\eta_{5}^{2}\right)\right] \\
& +\frac{C_{11223}}{4}\left(\eta_{1}^{2} \eta_{2}^{2} \eta_{3}+\eta_{1}^{2} \eta_{3}^{2} \eta_{2}+\eta_{3}^{2} \eta_{2}^{2} \eta_{1}\right)+\frac{C_{11244}}{4}\left[\eta_{1}^{2} \eta_{4}^{2}\left(\eta_{2}+\eta_{3}\right)+\eta_{2}^{2} \eta_{5}^{2}\left(\eta_{1}+\eta_{3}\right)+\eta_{3}^{2} \eta_{6}^{2}\left(\eta_{1}+\eta_{2}\right)\right] \\
& +\frac{C_{11255}}{4}\left[\eta_{1}^{2}\left(\eta_{2} \eta_{5}^{2}+\eta_{3} \eta_{6}^{2}\right)+\eta_{2}^{2}\left(\eta_{1} \eta_{4}^{2}+\eta_{3} \eta_{6}^{2}\right)+\eta_{3}^{2}\left(\eta_{1} \eta_{4}^{2}+\eta_{2} \eta_{5}^{2}\right)\right] \\
& +\frac{C_{11266}}{2}\left[\eta_{1} \eta_{2} \eta_{6}^{2}\left(\eta_{1}+\eta_{2}\right)+\eta_{1} \eta_{3} \eta_{5}^{2}\left(\eta_{1}+\eta_{3}\right)+\eta_{2} \eta_{3} \eta_{4}^{2}\left(\eta_{2}+\eta_{3}\right)\right] \\
& +\frac{C_{11456}}{2} \eta_{4} \eta_{5} \eta_{6}\left(\eta_{1}^{2}+\eta_{2}^{2}+\eta_{3}^{2}\right)+\frac{C_{12344}}{2} \eta_{1} \eta_{2} \eta_{3}\left(\eta_{4}^{2}+\eta_{5}^{2}+\eta_{6}^{2}\right) \\
& +C_{12456} \eta_{4} \eta_{5} \eta_{6}\left(\eta_{1} \eta_{2}+\eta_{1} \eta_{3}+\eta_{2} \eta_{3}\right)+\frac{C_{14444}}{24}\left(\eta_{1} \eta_{4}^{4}+\eta_{2} \eta_{5}^{4}+\eta_{3} \eta_{6}^{4}\right) \\
& +\frac{C_{14455}}{4}\left[\eta_{4}^{2} \eta_{5}^{2}\left(\eta_{1}+\eta_{2}\right)+\eta_{4}^{2} \eta_{6}^{2}\left(\eta_{1}+\eta_{3}\right)+\eta_{5}^{2} \eta_{6}^{2}\left(\eta_{2}+\eta_{3}\right)\right] \\
& +\frac{C_{15555}}{24}\left[\eta_{5}^{4}\left(\eta_{1}+\eta_{3}\right)+\eta_{4}^{4}\left(\eta_{2}+\eta_{3}\right)+\eta_{6}^{4}\left(\eta_{1}+\eta_{2}\right)\right] \\
& +\frac{C_{15566}}{2}\left(\eta_{1} \eta_{5}^{2} \eta_{6}^{2}+\eta_{2} \eta_{4}^{2} \eta_{6}^{2}+\eta_{3} \eta_{4}^{2} \eta_{5}^{2}\right)+\frac{C_{44456}}{2} \eta_{4} \eta_{5} \eta_{6}\left(\eta_{4}^{2}+\eta_{5}^{2}+\eta_{6}^{2}\right) .
\end{aligned}
$$

Equations (A7) to (A9) give the resulting expressions for the elastic energy density [Eqn. (A5)] for the third, fourth, and fifth order nonlinear elastic contributions. Apart from a few typographical errors in the earlier papers, the expressions are similar to those found in Refs. [42-44].

[1] M. Fallot and R. Hocart, Rev. Sci. 77, 498 (1939).

[2] J. S. Kouvel and C. C. Hartelius, J. Appl. Phys. 33, 1343 (1962).

[3] G. Shirane, R. Nathans, and C. W. Chen, Phys. Rev. 134, A1547 (1964).

[4] X. Marti, I. Fina, C. Frontera, J. Liu, P. Wadley, Q. He, R. J. Paull, J. D. Clarkson, J. Kudrnovský, I. Turek, J. Kuneš, D. Yi, J.-H. Chu, C. T. Nelson, L. You, E. Arenholz, S. Salahuddin, J. Fontcuberta, T. Jungwirth, and R. Ramesh, Nat. Mater. 13, 367 (2014).

[5] R. O. Cherifi, V. Ivanovskaya, L. C. Phillips, A. Zobelli, I. C. Infante, E. Jacquet, V. Garcia, S. Fusil, P. R. Briddon, N. Guiblin, A. Mougin, A. A. Ünal, F. Kronast, S. Valencia, B. Dkhil, A. Barthélémy, and M. Bibes, Nat. Mater. 13, 345 (2014).

[6] Y. Lee, Z. Q. Liu, J. T. Heron, J. D. Clarkson, J. Hong, C. Ko, M. D. Biegalski, U. Aschauer, S. L. Hsu, M. E. Nowakowski, J. Wu, H. M. Christen, S. Salahuddin, J. B. Bokor, N. A. Spaldin, D. G. Schlom, and R. Ramesh, Nat. Commun. 6, 5959 (2015).

[7] V. L. Moruzzi and P. M. Marcus, Phys. Rev. B 46, 2864 (1992).

[8] M. E. Gruner, E. Hoffmann, and P. Entel, Phys. Rev. B 67, 064415 (2003).

[9] S. Lounis, M. Benakki, and C. Demangeat, Phys. Rev. B 67, 094432 (2003).

[10] R. Y. Gu and V. P. Antropov, Phys. Rev. B 72, 012403 (2005).

[11] L. M. Sandratskii and P. Mavropoulos, Phys. Rev. B 83, 174408 (2011).
[12] A. Szajek and J. A. Morkowski, Physica B 193, 81 (1994).

[13] J. M. Lommel and J. S. Kouvel, J. Appl. Phys. 38, 1263 (1967).

[14] H. Miyajima and S. Yuasa, J. Magn. Magn. Mater. 104-107, 2025 (1992).

[15] M. Pugacheva, J. A. Morkowski, A. Jezierski, and A. Szajek, Solid State Commun. 92, 731 (1994).

[16] G. Kresse and J. Hafner, Phys. Rev. B 47, 558 (1993).

[17] G. Kresse and J. Hafner, Phys. Rev. B 49, 14251 (1994).

[18] G. Kresse and J. Furthmuller, Comput. Mater. Sci. 6, 15 (1996).

[19] G. Kresse and J. Furthmuller, Phys. Rev. B 54, 11169 (1996).

[20] J. P. Perdew, K. Burke, and M. Ernzerhof, Phys. Rev. Lett. 77, 3865 (1996).

[21] V. I. Anisimov, J. Zaanen, and O. K. Andersen, Phys. Rev. B 44, 943 (1991).

[22] S. L. Dudarev, G. A. Botton, S. Y. Savrasov, C. J. Humphreys, and A. P. Sutton, Phys. Rev. B 57, 1505 (1998).

[23] P. E. Blöchl, Phys. Rev. B 50, 17953 (1994).

[24] G. Kresse and D. Joubert, Phys. Rev. B 59, 1758 (1999).

[25] A. Togo, F. Oba, and I. Tanaka, Phys. Rev. B 78, 134106 (2008).

[26] G. Shirane, C. W. Chen, P. A. Flinn, and R. Nathans, Phys. Rev. 131, 183 (1963).

[27] B. K. Ponomarev, Sov. Phys. JETP 36, 105 (1973).

[28] M. J. Mehl, B. M. Klein, and D. A. Papaconstantopoulos, in First-Principles Calculations of Elastic Properties of Metals, 
edited by J. H. Westbrook and R. L. Fleischer, Intermetallic Compounds: Principles (John Wiley and Sons, London, 1994).

[29] D. W. Cooke, F. Hellman, C. Baldasseroni, C. Bordel, S. Moyerman, and E. E. Fullerton, Phys. Rev. Lett. 109, 255901 (2012).

[30] R. J. Zeches, M. D. Rossell, J. X. Zhang, A. J. Hatt, Q. He, C.-H. Yang, A. Kumar, C. H. Wang, A. Melville, C. Adamo, G. Sheng, Y.-H. Chu, J. F. Ihlefeld, R. Erni, C. Ederer, V. Gopalan, L. Q. Chen, D. G. Schlom, N. A. Spaldin, L. W. Martin, and R. Ramesh, Science 326, 977 (2009).

[31] S. Mankovsky, S. Polesya, K. Chadova, H. Ebert, J. B. Staunton, T. Gruenbaum, M. A. W. Schoen, C. H. Back, X. Z. Chen, and C. Song, arXiv:1606.02072.

[32] G. Grimvall, B. Magyari-Köpe, V. Ozolinšs, and K. A. Persson, Rev. Mod. Phys. 84, 945 (2012).

[33] F. D. Murnaghan, Proc. Natl. Acad. Sci. USA 30, 244 (1944).
[34] F. Birch, Phys. Rev. 71, 809 (1947).

[35] A. Corana, M. Marchesi, C. Martini, and S. Ridella, ACM Transactions on Mathematical Software (TOMS) 13, 262 (1987).

[36] H. C. Herper, E. Hoffmann, and P. Entel, Phys. Rev. B 60, 3839 (1999).

[37] G. Y. Guo and H. H. Wang, Chin. J. Phys. 38, 949 (2000).

[38] S. L. Dudarev and P. M. Derlet, J. Phys.: Condens. Matter 17, 7097 (2005).

[39] D. J. Dunstan, S. H. B. Bosher, and J. R. Downes, Appl. Phys. Lett. 80, 2672 (2002).

[40] A. S. Johal and D. J. Dunstan, Phys. Rev. B 73, 024106 (2006).

[41] M. Łopuszyński and J. A. Majewski, Phys. Rev. B 76, 045202 (2007).

[42] P. B. Ghate, J. Appl. Phys. 35, 337 (1964).

[43] D. Y. Chung and Y. Li, Acta Crystallogr., Sect. A 30, 1 (1974).

[44] H. Wang and M. Li, Phys. Rev. B 79, 224102 (2009). 